\title{
Conversion therapy for pancreatic cancer with peritoneal metastases using intravenous and intraperitoneal paclitaxel with S-1
}

\author{
HIROMITSU KITAYAMA ${ }^{1}$, YASUSHI TSUJI ${ }^{1}$, TOMOHIRO KONDO ${ }^{1}$, JUNKO SUGIYAMA ${ }^{1}$, \\ MICHIAKI HIRAYAMA ${ }^{2}$, KAZUYUKI YAMAMOTO ${ }^{3}$, YOU KAWARADA ${ }^{3}$, \\ YUMIKO OYAMADA $^{4}$ and SATOSHI HIRANO ${ }^{5}$
}

\author{
Departments of ${ }^{1}$ Medical Oncology, ${ }^{2}$ Gastroenterology, ${ }^{3}$ Surgery and ${ }^{4}$ Surgical Pathology, Tonan Hospital, Sapporo, \\ Hokkaido 060-0001; ${ }^{5}$ Department of Surgical Oncology, Hokkaido University \\ Graduate School of Medicine, Sapporo, Hokkaido 060-0808, Japan
}

Received March 24, 2016; Accepted September 23, 2016

DOI: $10.3892 / \mathrm{mco} .2016 .1051$

\begin{abstract}
Combination chemotherapy consisting of systemic and intraperitoneal agents against peritoneal metastases from several types of cancer has shown promising results. We herein report a case in which combination therapy with intravenous and intraperitoneal paclitaxel with S-1 converted an unresectable pancreatic cancer with peritoneal metastases into a resectable one. The patient was a 65 -year old woman with recurrent pancreatitis for 5 months. Endoscopic ultrasonography-guided fine-needle aspiration revealed minute epithelial masses composed of cells with irregular nuclei in the pancreatic body. The patient underwent abdominal surgery, but no excision was performed, as two peritoneal metastases in the bursa omentalis were detected. Combination therapy was initiated, consisting of intravenous and intraperitoneal paclitaxel with S-1 as a single-center clinical trial. The regimen consisted with 2-week administration of S-1 (80 mg per day) followed by 1 week of rest, intravenous paclitaxel $50 \mathrm{mg} / \mathrm{m}^{2}$, and intraperitoneal paclitaxel $20 \mathrm{mg} / \mathrm{m}^{2}$ by a peritoneal access device on days 1 and 8 . Over the seven cycles of the chemotherapy, the primary lesion did not change in size, and peritoneal lavage cytology remained negative. After confirming the disappearance of the peritoneal lesions by exploratory laparoscopy, the patient underwent distal pancreatectomy combined with resection of the transverse mesocolon and stomach wall. Thus, the 2-way
\end{abstract}

Correspondence to: Dr Hiromitsu Kitayama, Department of Medical Oncology, Tonan Hospital, Kita 1-jo Nishi 6-chome, Chuo-ku, Sapporo, Hokkaido 060-0001, Japan

E-mail: m02032hk@jichi.ac.jp

Abbreviations: PTX, paclitaxel; IV, intravenous; IP, intraperitoneal

Key words: case report, regional perfusion chemotherapy, pancreatic cancer, peritoneal metastasis, intraperitoneal paclitaxel, $\mathrm{S}-1$, conversion, complete response chemotherapy of intravenous and intraperitoneal paclitaxel with S-1 was well-tolerated and was able to convert pancreatic cancer with peritoneal metastases to resectable disease.

\section{Introduction}

The prognosis of pancreatic cancer patients with peritoneal metastases remains very poor, as peritoneal metastases are one of the major life-threatening factors in such patients $(1,2)$. S-1, an oral 5-fluorouracil (5-FU) derivative and paclitaxel (PTX) have been proven to be effective in the treatment of metastatic pancreatic cancer $(3,4)$. Combination chemotherapy consisting of intravenous (IV) and intraperitoneal (IP) PTX with S-1 was well-tolerated and achieved promising results against peritoneal metastases from gastric and pancreatic cancer $(5,6)$. However, to the best of our knowledge, a conversion of an unresectable pancreatic cancer with peritoneal metastases to a resectable one by this combination chemotherapy has not yet been reported.

We herein present a case of clinical complete response to IV and IP PTX with S-1 for peritoneal metastases from pancreatic cancer. This combination chemotherapy was able to convert an unresectable pancreatic cancer with peritoneal metastases into resectable disease.

\section{Case report}

The patient was a 65 -year-old Japanese woman, presenting with intermittent constipation, abdominal distention and back pain. The patient was a non-drinker and a non-smoker. Laboratory examinations revealed increased levels of pancreatic enzymes in the blood and urine. The patient was initially diagnosed with acute pancreatitis and was repeatedly hospitalized; she was referred to Tonan Hospital (Sapporo, Japan), as her symptoms had continued for 5 months in 2013. Computed tomography and magnetic resonance cholangiopancreatography revealed dilation of the tail of the main pancreatic duct, which was consistent with post-inflammatory changes. Tumor markers, including carcinoembryonic antigen and carbohydrate antigen 
Table I. Timeline of interventions and outcomes.

Month, year

July, 2012

January, 2013

July, 2013

August, 2013

January, 2014

February, 2014

September, 2014

February, 2015
Event

Onset of intermittent constipation, abdominal distention and back pain.

Diagnosis of pancreatic body cancer causing recurrent acute pancreatitis.

Exploratory open abdominal surgery revealing two peritoneal metastases.

Implantation of a peritoneal access device.

Seven cycles of intraperitoneal and intravenous paclitaxel with S-1.

Exploratory laparoscopy revealing malignancy-negative peritoneal white nodules.

Distal pancreatectomy as radical resection.

Chemotherapy resumed as adjuvant therapy.

Peritoneal recurrence in Douglas pouch.

Death from disease.
19-9, were normal. Endoscopic ultrasonography revealed poorly marginated low-density lesion in the pancreatic body and fine-needle aspiration was subsequently performed. Histological examination revealed minute epithelial masses composed of cells with irregular nuclei. The patient underwent open abdominal surgery due to suspected pancreatic body cancer in June, 2013. However, as two nodules in the bursa omentalis were found during the surgery and were diagnosed as tubular adenocarcinoma, pancreatectomy was abandoned (Fig. 1). Combination therapy was initiated, consisting of IV and IP PTX with S-1 (Taiho Pharmaceutical Co., Ltd., Tokyo, Japan), as a single-center clinical trial in August, 2013. A peritoneal access device was implanted in the right lower abdominal wall, and an indwelling catheter was placed in the pelvic cavity. The chemotherapy regimen was as follows: 2-week administration of S-1 (80 mg per day) followed by a 1-week rest, with IV PTX $50 \mathrm{mg} / \mathrm{m}^{2}$ and IP PTX $20 \mathrm{mg} / \mathrm{m}^{2}$ on days 1 and 8 . The primary lesion did not change in size and peritoneal lavage cytology remained negative during seven cycles of chemotherapy. An exploratory laparoscopy was performed and peritoneal white nodules were identified, which were found to be negative for malignancy on frozen section biopsy in January, 2014. Subsequently, distal pancreatectomy was performed, combined with resection of the transverse mesocolon and stomach wall (Fig. 2). The postoperative course was uneventful.

The pancreatic tumor was for the most part replaced by extensive fibrosis (Fig. 3A). Furthermore, fibrosis and xanthogranulomatous reaction with extensive fibrosis were observed in the pancreatic capsule and transverse mesocolon, indicating that almost all peritoneal metastases were eliminated after the chemotherapy; only one nodule in the gastropancreatic fold was microscopically diagnosed as residual metastasis (Fig. 3B). The histopathological diagnosis was ductal carcinoma, ypT3, ypN1, ypM1 (PER), G2, L0, V1, R0, ypStage IV according to the 7th edition of the American Joint Committee on Cancer/International Union Against Cancer TNM classification (https://cancerstaging.org/references-tools/quickreferences/Documents/PancreasSmall.pdf). The effect of the preoperative chemotherapy was classified as grade II using the Evans classification (7).

The patient continued to receive the same chemotherapy in the adjuvant setting for 8 months after the surgery, with minor hair and appetite loss. However, peritoneal carcinoma appeared again in the Douglas pouch in September, 2014. Local recurrence or other metastases were not detected at the time of recurrence. The patient succumbed to the disease 6 months later, in February, 2015 (Table I).

\section{Discussion}

The course of this patient draws attention to two important clinical issues as follows: The first point is that the 2-way chemotherapy combining IV and IP PTX with S-1 was able to achieve clinical complete response against peritoneal metastases from pancreatic cancer. The ability of IP PTX to maintain high concentrations in the peritoneal cavity may have contributed to the strong antitumor effect against the peritoneal metastases, and the tumor responded well to the drug combination with S-1. IP administration of PTX was aimed at acting directly on the peritoneal metastases at a high concentration. When PTX is administered IP, it is absorbed slowly due to its high molecular weight and lipophilic properties, and IP PTX itself is considered to be associated with a relatively mild toxicity (8). IP administration of PTX has been used together with systemic chemotherapy for peritoneal metastases from ovarian cancer, with a significant survival benefit (9). Additionally, S-1 is one of the oral 5-FU agents that were found to be non-inferior to gemcitabine in terms of overall survival in East-Asian patients with metastatic pancreatic cancer (3). Yamaguchi et al reported that IV and IP PTX with S-1 was well tolerated and highly effective against peritoneal metastases from gastric cancer (5), and the MPACT trial demonstrated that PTX was effective in metastatic pancreatic cancer (4). In fact, IV and IP PTX with S-1 recently exhibited promising results in gemcitabine-refractory pancreatic cancer (6). On pathological examination in our case, almost all peritoneal metastases appeared to have been eradicated by chemotherapy.

The second important point is that this combination chemotherapy converted unresectable pancreatic cancer with peritoneal metastases into resectable disease. Conversion therapy, which is defined as therapy achieving transformation of unresectable to resectable disease, has been established in colon cancer management (10). However, the viability of conversion therapy for other types of cancer has not been 


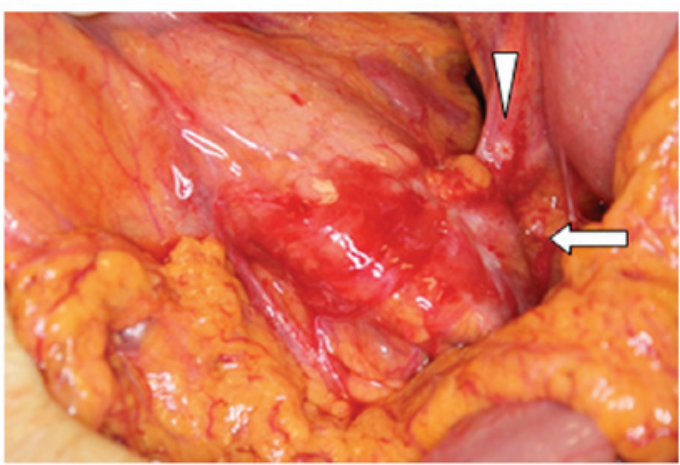

Figure 1. Intraoperative findings prior to chemotherapy. The tumor was located in the pancreatic tail (arrow) and one of two metastases in the bursa omentalis was located in gastropancreatic fold (arrowhead).
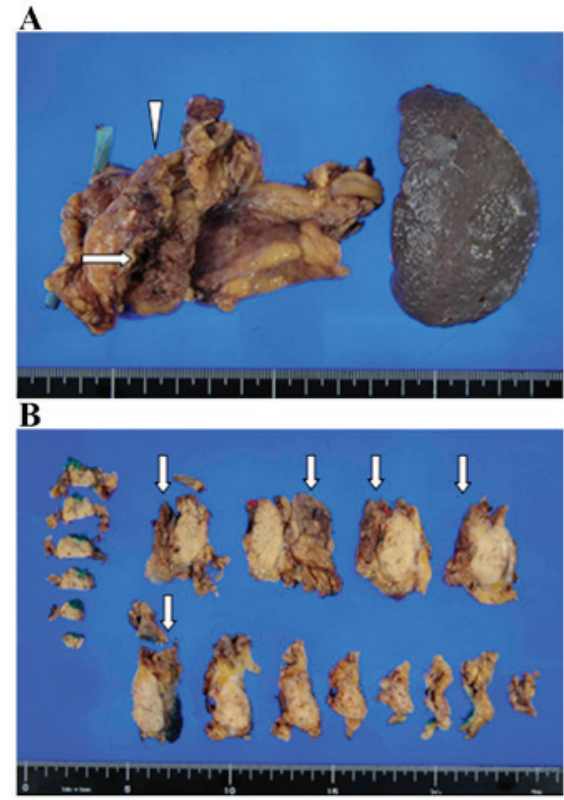

Figure 2. Macroscopic images of formalin-fixed specimen obtained from distal pancreatectomy with splenectomy. (A) The specimen included the gastropancreatic fold, stomach wall (arrow) and transverse mesocolon (arrowhead), where peritoneal metastases were found. The stumps of the stomach wall and pancreas were marked by blue and green ink, respectively. (B) Dissected specimens showing the distribution of the white solid tumor (arrows, stomach wall).

sufficiently investigated. If chemotherapy manages to control peritoneal metastases over a long time period, primary tumor resection may prolong patient overall survival. Conversion therapy for gastric cancer has been considered beneficial for patients receiving tolerable and satisfactory chemotherapy, as improved new regimens including systemic and IP chemotherapy may achieve long-term control of the metastatic disease in certain cases (11). This may be also applicable to pancreatic cancer. It was recently reported that 3 patients with pancreatic cancer with macroscopic peritoneal metastases underwent R0 resection of the primary tumor following treatment with IV and IP PTX with S-1 (6). Those 3 cases, as well as our patient, had residual cancer cells in the primary lesion after chemotherapy. Primary tumor resection after chemotherapy may be preferable if peritoneal metastases

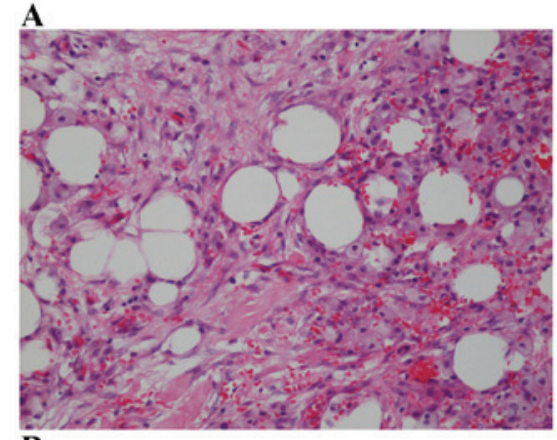

B

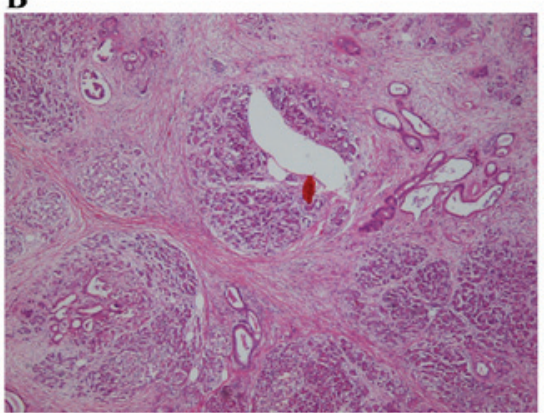

Figure 3. Microscopic images of the pancreatic tumor stained with hematoxylin-eosin. (A) The tumor in the pancreatic tissue (magnification, $\mathrm{x} 1,000$ ) and (B) one peritoneal metastasis in the gastropancreatic fold (magnification, $\mathrm{x} 400$ ) exhibited extensive fibrosis and xanthogranulomatous reaction with aggregated foamy macrophage clusters, indicating disappearance of peritoneal metastases following combination chemotherapy.

are controlled well over a long time period. Our patient received the combination chemotherapy for 5 months before primary tumor resection. Distal pancreatectomy following chemotherapy was not complicated. Thus, combination therapy consisting of IV and IP PTX with S-1 was effective in controlling peritoneal metastases from pancreatic cancer and convert the disease status to resectable.

Longer duration of chemotherapy ( $>8$ months prior to surgery) may be preferable as conversion therapy. Unfortunately, the disease recurred at the Douglas pouch 8 months after surgery, which was likely due to drug resistance of the malignant cells. In a phase II study, all 8 pancreatic cancer patients with peritoneal metastases received the combination chemotherapy for $>8$ months prior to surgery (6). Additionally, a Japanese multicenter retrospective study demonstrated that preoperative treatment for $>8$ months was favorable in terms of overall survival for initially unresectable pancreatic cancer (12).

In conclusion, the 2-way chemotherapy consisting of IV and IP PTX with S-1 converted unresectable pancreatic cancer with peritoneal metastases into resectable disease and this combination chemotherapy achieved clinical complete response against the peritoneal metastases with good tolerance. IP PTX has the ability to maintain high concentrations for prolonged periods of time, and this combination chemotherapy has the potential to become a conversion therapy for pancreatic cancer with peritoneal metastases. However, further investigation is required to evaluate the substantivity of this treatment and to determine whether the pancreatic tumor should be resected after a satisfactory response of the peritoneal metastases to chemotherapy. 


\section{Acknowledgements}

The authors would like to thank the patient for her kind cooperation, including permission to publish the details of this case, and Mr. David Hochman for reviewing the language of our article. Taiho Pharmaceutical Co., Ltd. provided lecture fees to Dr Kitayama and a gratitude scholarship to Professor Hirano. Ono Pharmaceutical Co., Ltd. provided lecture fees to Dr Tsuji. The present study was approved by the Institutional Review Board of Tonan Hospital.

\section{References}

1. DeWitt J, Yu M, Al-Haddad MA, Sherman S, McHenry L and Leblanc JK: Survival in patients with pancreatic cancer after the diagnosis of malignant ascites or liver metastases by EUS-FNA. Gastrointest Endosc 71: 260-265, 2010.

2. Morizane C, Okusaka T, Morita S, Tanaka K, Ueno H, Kondo S, Ikeda M, Nakachi $\mathrm{K}$ and Mitsunaga S: Construction and validation of a prognostic index for patients with metastatic pancreatic adenocarcinoma. Pancreas 40: 415-421, 2011.

3. Ueno H, Ioka T, Ikeda M, Ohkawa S, Yanagimoto H, Boku N, Fukutomi A, Sugimori K, Baba H, Yamao K, et al: Randomized phase III study of gemcitabine plus S-1, S-1 alone, or gemcitabine alone in patients with locally advanced and metastatic pancreatic cancer in Japan and Taiwan: GEST study. J Clin Oncol 31: 1640-1648, 2013.

4. Von Hoff DD, Ervin T, Arena FP, Chiorean EG, Infante J, Moore M, Seay T, Tjulandin SA, Ma WW, Saleh MN, et al: Increased survival in pancreatic cancer with nab-paclitaxel plus gemcitabine. N Engl J Med 369: 1691-1703, 2013.
5. Yamaguchi H, Kitayama J, Ishigami H,Emoto S, Yamashita H and Watanabe T: A Phase 2 trial of Intravenous and intraperitoneal paclitaxel combined with S-1 for treatment of gastric cancer with macroscopic peritoneal metastasis. Cancer 119: 3354-3358, 2013.

6. Satoi S, Fujii T, Yanagimoto H, Motoi F, Kurata M, Takahara N, Yamada S, Yamamoto T, Mizuma M, Honda G, et al: Multicenter Phase II study of intravenous and intraperitoneal paclitaxel with S-1 for pancreatic ductal adenocarcinoma patients with peritoneal metastasis. Ann Surg: Mar 11, 2016 (Epub ahead of print).

7. Evans DB, Rich TA, Byrd DR, Cleary KR, Connelly JH, Levin B, Charnsangavej C, Fenoglio CJ and Ames FC: Preoperative chemoradiation and pancreaticoduodenectomy for adenocarcinoma of the pancreas. Arch Surg 127: 1335-1339, 1992.

8. Gelderblom H, Verweij J, van Zomeren DM, Buijs D, Ouwens L, Nooter K, Stoter G and Sparreboom A: Influence of Cremophor $\mathrm{El}$ on the bioavailability of intraperitoneal paclitaxel. Clin Cancer Res 8: 1237-1241, 2002.

9. Armstrong DK, Bundy B, Wenzel L, Huang HQ, Baergen R, Lele S, Copeland LJ, Walker JL and Burger RA; Gynecologic Oncology Group: Intraperitoneal cisplatin and paclitaxel in ovarian cancer. N Engl J Med 354: 34-43, 2006.

10. Power DG and Kemeny NE: Chemotherapy for the conversion of unresectable colorectal cancer liver metastases to resection. Crit Rev Oncol Hematol 79: 251-264, 2011

11. Yoshida K, Yamaguchi K, Okumura N, Tanahashi T and Kodera Y: Is conversion therapy possible in stage IV gastric cancer: The proposal of new biological categories of classification. Gastric Cancer 19: 329-338, 2016.

12. Satoi S, Yamaue H, Kato K, Takahashi S, Hirono S, Takeda S, Eguchi H, Sho M, Wada K, Shinchi H, et al: Role of adjuvant surgery for patients with initially unresectable pancreatic cancer with a long-term favorable response to non-surgical anti-cancer treatments: Results of a project study for pancreatic surgery by the Japanese society of Hepato-Biliary-Pancreatic surgery. J Hepatobiliary Pancreat Sci 20: 590-600, 2013. 